\title{
Cálculo das Frequências e dos Modos de Vibração de um Sistema de Duas Vigas Acopladas
}

\author{
Aline Brum Seibel \\ Programa de Pós-Graduação em Matemática, UFSM \\ 97105-900, Santa Maria, RS \\ E-mail: alinebrumseibel@gmail.com,

\section{Rosemaira Dalcin Copetti} \\ UFSM - Departamento de Matemática \\ Cidade Universitária \\ 97105-900, Santa Maria, RS \\ E-mail: rmaira@smail.ufsm.br.
}

\begin{abstract}
Resumo: Neste trabalho é realizado um estudo sobre vibrações livres e forçadas de um sistema composto por duas vigas Euler-Bernoulli acopladas. As vigas são paralelas, de mesmo comprimento, simplesmente apoiadas e conectadas por uma camada viscoelástica. O estudo é realizado através da análise modal e de uma formulação matricial em blocos, onde os modos de vibração do sistema são escritos em termos da base dinâmica, obtida através da solução dinâmica de uma equação diferencial de quarta ordem. É considerado o amortecimento de Rayleigh e o sistema é desacoplado usando o teorema dos modos normais. A resposta forçada é escrita em termos da resposta impulso matricial.
\end{abstract}

Palavras-chave: Viga Dupla Euler-Bernoulli, Frequências, Modos de Vibração, Resposta Impulso Matricial, Amortecimento de Rayleigh.

\section{Introdução}

Estruturas do tipo viga são amplamente utilizadas em muitos ramos da engenharia civil, mecânica e aeroespacial. Em Abu-Hilal [1] a resposta dinâmica de um sistema de duas vigas EulerBernoulli considerando uma carga constante em movimento é estudado. As duas vigas são conectadas por uma camada viscoelástica e o sistema é dasacoplado através de uma equação que inclui a deflexão da primeira e segunda viga. No trabalho de Oniszczuk [8] vibrações livres de um sistema de viga dupla simplesmente apoiadas conectadas continuamente por uma camada elástica são consideradas, onde o movimento do sistema é descrito por uma equação diferencial de quarta ordem, a qual é resolvida pelo método de Bernoulli-Fourier. Vu, em [10], apresenta um método exato para determinar a resposta forçada de um sistema de viga dupla submetido a uma excitação harmônica, onde a resposta livre é obtida para diferentes condições de contorno. Neste trabalho, é considerado um sistema de duas vigas acopladas sujeito a uma força externa. O sistema consiste de duas vigas do tipo Euler-Bernoulli, paralelas, de mesmo comprimento acopladas por uma camada viscoelástica, a qual é composta por um sistema mola-amortecedor. A análise modal, uma formulação matricial em blocos e a base dinâmica $[4,3]$, são utilizadas para determinar as frequências naturais e os modos de vibração do sistema. Os modos de vibração são escritos usando a resposta fundamental para compor a base de soluções. No cálculo da resposta forçada é considerado o amortecimento de Rayleigh e a ortogonalidade dos modos usada para desacoplar o sistema envolvido. O problema livre e forçado sem amortecimento foi considerado em [9]. 


\section{Formulação do Problema}

Considere o sistema dado pela Figura 1 , onde $v_{i}(t, x)$ é o deslocamento da viga $i$, para $i=1,2$. O modelo matemático baseado na teoria de Euler-Bernoulli para vigas é dado por [1]:

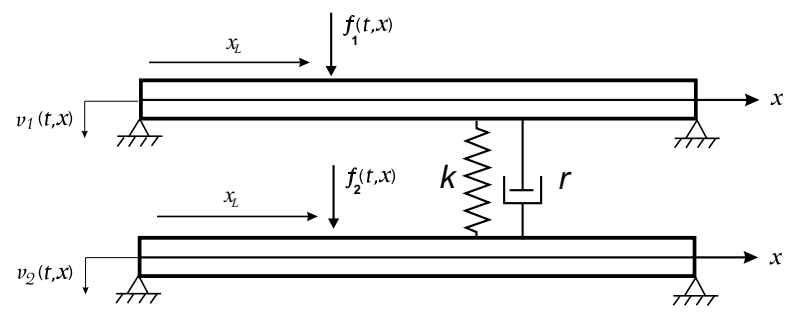

Figura 1: Sistema de viga dupla com amortecimento sujeito a uma força externa

$$
\begin{aligned}
& \rho_{1} A_{1} \frac{\partial^{2} v_{1}}{\partial t^{2}}+k\left(v_{1}-v_{2}\right)+c_{1} \frac{\partial v_{1}}{\partial t}+r\left(\frac{\partial v_{1}}{\partial t}-\frac{\partial v_{2}}{\partial t}\right)+E_{1} I_{1} \frac{\partial^{4} v_{1}}{\partial x^{4}}=f_{1}(t, x), \\
& \rho_{2} A_{2} \frac{\partial^{2} v_{2}}{\partial t^{2}}+k\left(v_{2}-v_{1}\right)+c_{2} \frac{\partial v_{2}}{\partial t}+r\left(\frac{\partial v_{2}}{\partial t}-\frac{\partial v_{1}}{\partial t}\right)+E_{2} I_{2} \frac{\partial^{4} v_{2}}{\partial x^{4}}=f_{2}(t, x),
\end{aligned}
$$

onde $E_{i}$ denota o módulo de elasticidade de Young, $I_{i}$ o momento de inércia, $\rho_{i}$ a densidade linear de massa e $A_{i}$ a área da seção transversal da viga $i$. As constantes de rigidez de mola $k$ e a constante de amortecimento $r$, compõem a camada viscoelástica, $c_{i}$ denota a constante de amortecimento de cada viga, $f_{i}$ a força externa, $v_{i}(t, x)$ a deflexão transversal da viga no instante $t$ na posição $x$, onde $i=1$ indica a primeira viga e $i=2$ a segunda viga.

As condições de contorno para o sistema de viga dupla simplesmente apoiado são:

$$
v_{i}(t, 0)=0, \quad v_{i}(t, L)=0, \quad v_{i}^{\prime \prime}(t, 0)=0, \quad v_{i}^{\prime \prime}(t, L)=0,
$$

onde $v_{i}^{\prime \prime}=\frac{\partial^{2} v_{i}}{\partial x^{2}}, i=1,2$.

Matricialmente, as equações (1) e (2) podem ser escritas na forma

$$
\mathbf{M} \frac{\partial^{2} \mathbf{v}}{\partial t^{2}}+\mathbf{C} \frac{\partial \mathbf{v}}{\partial t}+\mathbf{K} \mathbf{v}=\mathbf{F}(t, x)
$$

onde

$$
\mathbf{M}=\left[\begin{array}{cc}
\rho_{1} A_{1} & 0 \\
0 & \rho_{2} A_{2}
\end{array}\right], \mathbf{C}=\left[\begin{array}{cc}
c_{1}+r & -r \\
-r & c_{2}+r
\end{array}\right], \quad \mathbf{K}=\left[\begin{array}{cc}
E_{1} I_{1} \frac{\partial^{4}}{\partial x^{4}}+k & -k \\
-k & E_{2} I_{2} \frac{\partial^{4}}{\partial x^{4}}+k
\end{array}\right],
$$

$\mathbf{F}=\left[f_{1}(t, x) f_{2}(t, x)\right]^{T}$ é o vetor força externa e $\mathbf{v}=\left[v_{1}(t, x) v_{2}(t, x)\right]^{T}$ a deflexão transversal.

\section{Resposta Livre}

Considerando o sistema homogêneo, $\mathbf{F}=\mathbf{0}$ em (4), sua solução pode ser escrita na forma

$$
\mathbf{v}(t, x)=e^{\lambda t} \mathbf{V}(x),
$$

onde $\mathbf{V}(x)=\left[V_{1}(x) V_{2}(x)\right]^{T}$ são as autofunções e $\lambda$ é um autovalor. Substituindo (6) em (4), obtém-se o problema de autovalor

$$
\mathbf{K}_{0} V^{(i v)}(x)+\left(\lambda^{2} \mathbf{M}+\lambda \mathbf{C}+\mathbf{K}_{k}\right) V(x)=\mathbf{0},
$$

onde $\mathbf{0}$ é a matriz nula de ordem 2, as matrizes $\mathbf{M}$ e $\mathbf{C}$ são dadas em (5) e

$$
\mathbf{K}_{0}=\left[\begin{array}{cc}
E_{1} I_{1} & 0 \\
0 & E_{2} I_{2}
\end{array}\right], \quad \mathbf{K}_{k}=\left[\begin{array}{cc}
k & -k \\
-k & k
\end{array}\right] .
$$


Das condições de contorno dadas em (3) e usando a solução (6), obtemos as condições de contorno para a equação modal (7)

$$
\mathbf{V}(0)=\mathbf{V}(L)=0 \text { e } \mathbf{V}^{\prime \prime}(0)=\mathbf{V}^{\prime \prime}(L)=0 .
$$

A solução geral de (7), pode ser obtida através do uso da base dinâmica, a qual é composta pela matriz $\mathbf{h}(x)$ de ordem 2 , solução do problema de valor inicial

$$
\begin{gathered}
\mathbf{K}_{0} \mathbf{h}^{(i v)}(x)+\left(\lambda^{2} \mathbf{M}+\lambda \mathbf{C}+\mathbf{K}_{k}\right) \mathbf{h}(x)=\mathbf{0} \\
\mathbf{h}(0)=\mathbf{0}, \mathbf{h}^{\prime}(0)=\mathbf{0}, \mathbf{h}^{\prime \prime}(0)=\mathbf{0}, \mathbf{K}_{0} \mathbf{h}^{\prime \prime \prime}(0)=\mathbf{I},
\end{gathered}
$$

e suas três primeiras derivadas $\mathbf{h}^{\prime}(x), \mathbf{h}^{\prime \prime}(x), \mathbf{h}^{\prime \prime \prime}(x)$, onde $\mathbf{0}$ é a matriz nula e $\mathbf{I}$ a matriz identidade, ambas de ordem 2. Assim, podemos escrever a solução de (7) da seguinte maneira:

$$
\mathbf{V}(x)=\mathbf{h}(x) \mathbf{e}_{1}+\mathbf{h}^{\prime}(x) \mathbf{e}_{2}+\mathbf{h}^{\prime \prime}(x) \mathbf{e}_{3}+\mathbf{h}^{\prime \prime \prime}(x) \mathbf{e}_{4}=\Phi(x) \mathbf{e}
$$

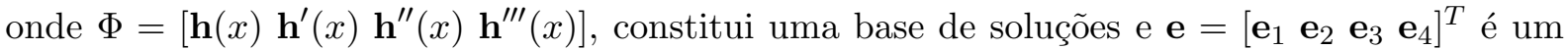
vetor constante $(8 \times 1)$ sendo $\mathbf{e}_{i}=\left[\begin{array}{ll}e_{i 1} & e_{i 2}\end{array}\right]^{T}$, para $i=1,2,3,4$.

Das condições de contorno (9), segue que $\mathbf{e}_{2}=\mathbf{e}_{4}=0$ em (11) e portanto a solução da equação (7) é simplificada para

$$
\mathbf{V}(x)=\mathbf{h}(x) \mathbf{e}_{1}+\mathbf{h}^{\prime \prime}(x) \mathbf{e}_{3} .
$$

Para o cálculo da base dinâmica usamos a fórmula fechada dada em [4], e os autovalores $\lambda$ são solução de $[5,3]$

$$
\operatorname{det}\left(\begin{array}{cc}
\mathbf{h}^{\prime \prime}(L) & \mathbf{h}^{\prime \prime \prime}(L) \\
\mathbf{h}^{\prime \prime \prime}(L) & \mathbf{h}^{(i v)}(L)
\end{array}\right)=0
$$

\section{Resposta Forçada}

A resposta forçada para o sistema matricial evolutivo dado em (4) pode ser escrita em termos da integral de convolução

$$
\mathbf{v}(t, x)=\int_{0}^{t} \int_{0}^{L} \mathbf{h}(t-\tau, x, \xi) \mathbf{F}(t, \xi) d \xi d \tau,
$$

onde $\mathbf{h}(t, x, \xi)$ é a resposta impulso matricial.

Como é bem conhecido na literatura [2], não é para qualquer matriz $\mathbf{C}$ que o sistema (4) é desacoplável. Uma condição suficiente para o desacoplamento é quando a matriz de amortecimento C é uma combinação linear das matrizes de massa e rigidez, conhecido como amortecimento de Rayleigh, ou seja:

$$
\mathbf{C}=\alpha \mathbf{M}+\beta \mathbf{K},
$$

onde $\alpha$ e $\beta$ são constantes reais e positivas. Substituindo (15) em (4) leva a:

$$
\mathbf{M} \ddot{\mathbf{v}}(t, x)+[\alpha \mathbf{M}+\beta \mathbf{K}] \dot{\mathbf{v}}(t, x)+\mathbf{K v}(t, x)=\mathbf{F}(t, x) .
$$

Para identificar a matriz $\mathbf{h}(t, x, \xi)$ em (14), a resposta forçada do sistema (4) pode ser escrita como, [7]

$$
\mathbf{v}(t, x)=\sum_{i=0}^{\infty} \mathbf{V}^{i}(x) \eta_{i}(t)
$$

onde $\eta_{i}(t)$ são coeficientes temporais a serem determinados e $\mathbf{V}^{i}(x)=\left[V_{1}^{i}(x) V_{2}^{i}(x)\right]^{T}$ são os modos de vibração do sistema não amortecido. Substituindo (17) em (16), multiplicando à 
esquerda a equação (16) por $\left(\mathbf{V}^{j}\right)^{T}(x)$, integrando de 0 a $L$ ambos os lados da equação e usando a ortogonalidade dos modos, obtém-se o sistema desacoplado

$$
\ddot{\eta}_{i}(t)+2 \zeta_{i} \omega_{i} \dot{\eta}_{i}(t)+\omega_{i}^{2} \eta_{i}(t)=G_{i}(t),
$$

sendo

$$
\zeta_{i}=\frac{1}{2} \beta \omega_{i}+\frac{1}{2 \omega_{i}} \alpha, \text { e } G_{i}(t)=\int_{0}^{L}\left(\mathbf{V}^{i}\right)^{T}(x) \mathbf{F}(t, x) d x
$$

e $\zeta_{i}$ é a relação de amortecimento e $\omega_{i}$ são as frequências naturais do sistema sem amortecimento. A solução geral da equação (18), considerando condições iniciais nulas e $0<\zeta_{i}<1$, é dada por

$$
\eta_{i}(t)=\int_{0}^{t} h_{i}(t-\tau) G_{i}(\tau) d \tau
$$

onde $h_{i}(t)$ é a resposta impulso temporal, solução do problema de valor inicial

$$
\begin{gathered}
\ddot{h}_{i}(t)+2 \zeta_{i} \omega_{i} \dot{h}_{i}(t)+\omega_{i}^{2} h_{i}(t)=0 \\
h_{i}(0)=0 \quad \dot{h}_{i}(0)=1,
\end{gathered}
$$

isto é, $h_{i}(t)=\frac{1}{\omega_{d}} e^{-\zeta_{i} \omega_{i} t} \sin \omega_{d} t$, e $\omega_{d}=\omega_{i} \sqrt{1-\zeta_{i}^{2}}$ é a frequência amortecida. Substituindo (20) em (17) e comparando com (14), temos que a resposta impulso matricial é dada por

$$
\mathbf{h}(t, x, \xi)=\sum_{i=1}^{\infty} h_{i}(t) \mathbf{V}^{i}(x)\left(\mathbf{V}^{i}\right)^{T}(\xi) .
$$

\section{Simulações}

Foram realizadas várias simulações. Apresentamos aqui, alguns resultados sem e com amortecimento. Para o caso amortecido, foi considerado o amortecimento na segunda viga. A resposta forçada foi obtida para uma força aplicada num ponto fixo $x=x_{L}$ da primeira viga, isto é,

$$
f_{1}(t, x)=f(t) \delta\left(x-x_{L}\right),
$$

onde $\delta$ é a função delta de Dirac e $x_{L}$ é um ponto qualquer da primeira viga para $0 \leq L \leq 10$, e $f_{2}(t, x)=0$. A resposta forçada em cada viga é dada por:

$$
\begin{aligned}
& v_{1}(t, x)=\int_{0}^{t} \int_{0}^{L} \mathbf{h}_{11} f(\tau) \delta\left(\xi-x_{L}\right) d \xi d \tau=\int_{0}^{t} f(\tau) \mathbf{h}_{11}\left(t-\tau, x, x_{L}\right) d \tau, \\
& v_{2}(t, x)=\int_{0}^{t} \int_{0}^{L} \mathbf{h}_{21} f(\tau) \delta\left(\xi-x_{L}\right) d \xi d \tau=\int_{0}^{t} f(\tau) \mathbf{h}_{21}\left(t-\tau, x, x_{L}\right) d \tau,
\end{aligned}
$$

onde $\mathbf{h}_{11}$ e $\mathbf{h}_{21}$ são componentes da matriz $\mathbf{h}(t, x, \xi)$, dada em (22).

Os parâmetros utilizados são: $L=10 \mathrm{~m}, E_{1}=E_{2}=1 \times 10^{10} \mathrm{~N} / \mathrm{m}^{-2}, \quad I_{1}=I_{2}=4 \times$ $10^{-4} \mathrm{~m}^{4}, \quad \rho_{1}=\rho_{2}=2 \times 10^{3} \mathrm{Kg} / \mathrm{m}^{-3}, A_{1}=A_{2}=5 \times 10^{-2} \mathrm{~m}^{2}, \quad k=1 \times 10^{5} \mathrm{~N} / \mathrm{m}^{2}, c_{1}=$ $1 N \mathrm{~ms}^{-1}, c_{2}=6 \times 10^{3} \mathrm{Nms}^{-1}, r=0, \alpha=0.5, \beta=0.01$, e o forçante $f(t)=P_{0} \cos (n t)$, sendo $P_{0}$ uma constante.

Na Tabela 1, apresentamos os quatro primeiros pares de autovalores do sistema para os dois casos. Cada par de autovalor é formado por $\lambda_{i 1}$ e $\lambda_{i 2}$ para $i=1,2,3,4$. Para o caso não amortecido, $\lambda_{i j}=\omega_{i j} I$, onde $\omega_{i j}$ é a frequência natural e $I$ a unidade imaginária.

Na Figura 2, apresentamos os dois primeiros modos de vibração, os quais são gerados pelos dois primeiros pares de autovalores. Podemos observar que os modos de vibração executam dois tipos de movimentos: síncrono e assíncrono, dependendo do autovalor considerado. Os autovalores $\lambda_{i 1}$ geram modos síncronos e os autovalores $\lambda_{i 2}$ geram modos assíncronos.

Na Figura 3, temos a resposta forçada na primeira viga para o forçante $f(t)=100 \cos \left(\frac{1}{10} t\right)$ aplicado no ponto $x=5$ e $x=9$. Na primeira coluna á apresentada a resposta forçada para $0 \leq x \leq 10$ e $0 \leq t \leq 1$, e na segunda coluna um corte em $x=5$ e $0 \leq t \leq 2$. 


\begin{tabular}{ccc}
\hline \hline$\lambda_{i j}$ & Caso não amortecido & Caso amortecido \\
\hline \hline$\lambda_{11}$ & $19.73920880 I$ & $-23.72950761+4.188071500 I$ \\
$\lambda_{12}$ & $48.88390703 I$ & $-6.275492393+39.55001988 I$ \\
$\lambda_{21}$ & $78.95683503 I$ & $-28.80093684+79.12363349 I$ \\
$\lambda_{22}$ & $90.74239282 I$ & $-1.204063078+85.08089084 I$ \\
$\lambda_{31}$ & $177.6530320 I$ & $-29.74180181+177.8903672 I$ \\
$\lambda_{32}$ & $183.1956337 I$ & $-0.2631945571+180.4459807 I$ \\
$\lambda_{41}$ & $315.8271071 I$ & $-29.91652844+315.9773581 I$ \\
$\lambda_{42}$ & $318.9765409 I$ & $-0.08852017015+317.4062843 I$ \\
\hline
\end{tabular}

Tabela 1: Quatro primeiros pares de autovalores do sistema sem e com amortecimento

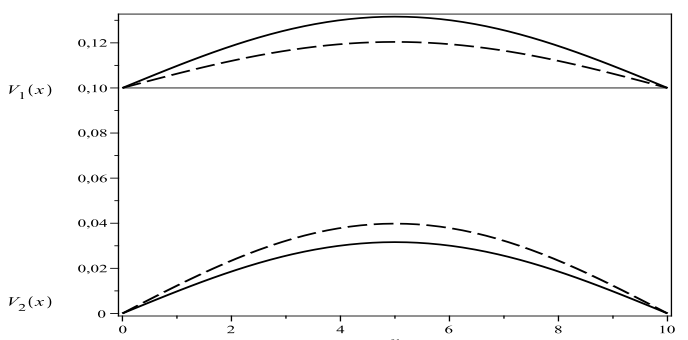

(a) Primeiro modo $\lambda_{11}$

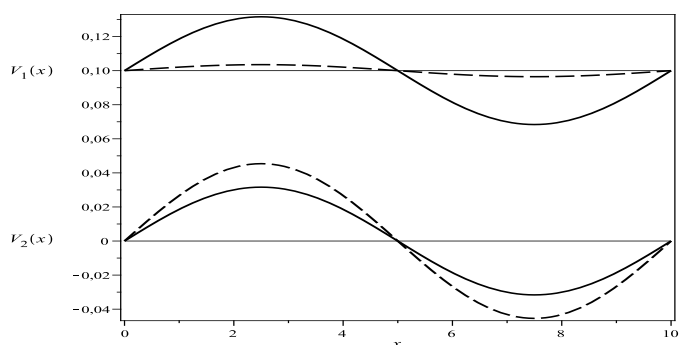

(c) Segundo modo $\lambda_{21}$

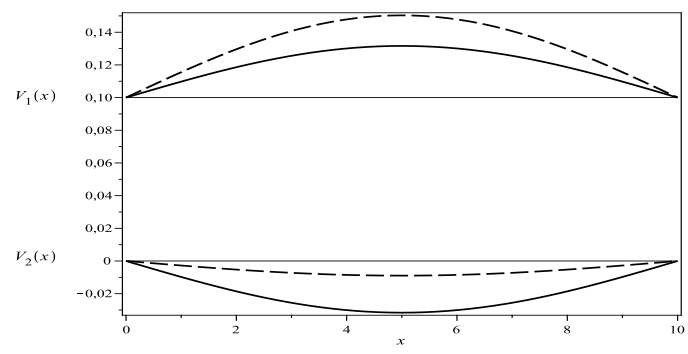

(b) Primeiro modo $\lambda_{12}$

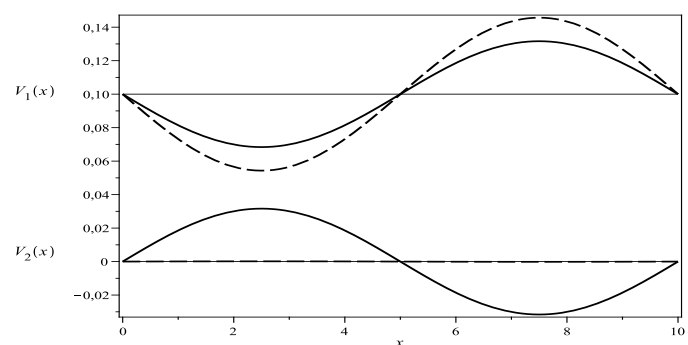

(d) Segundo modo $\lambda_{22}$

- Não amortecido - - Amortecido

Figura 2: Primeiros e segundos modos de vibração
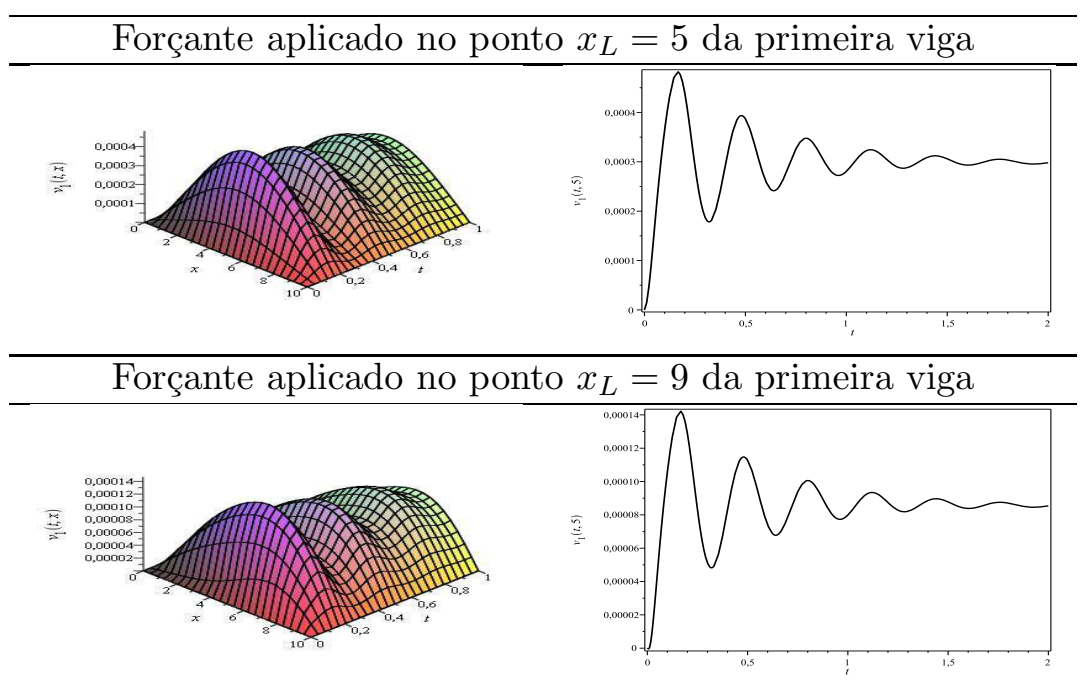

Figura 3: Resposta forçada com amortecimento para $f(t)=100 \cos \left(\frac{1}{10} t\right)$ 


\section{Conclusões}

Neste trabalho foi introduzida uma metodologia que usa a base dinâmica, a análise modal e uma formulação matricial em blocos para o estudo de vibrações livres e forçadas de um sistema de duas vigas acopladas elasticamente. $\mathrm{O}$ uso da base dinâmica facilita o cálculo das frequências e dos modos de vibração do problema acoplado, pois reduz a dimensão do problema. A resposta forçada do sistema com amortecimento de Rayleigh é escrita em termos da resposta impulso matricial. Essa metotodologia pode ser aplicada a vigas Timoshenko, vigas com condições de contorno clássicas e não-clássicas, vigas segmentadas, nanotubos de carbono. A contribuição do presente trabalho é a extensão dessa metodologia para o caso de vigas Euler-Bernoulli uniformes acopladas por uma camada viscoelástica.

\section{Referências}

[1] M. Abu-Hilal, Dynamic response of a double Euler-Bernoulli beam due to a moving constant load, J. Sound Vib., 297 (2006) 477-491.

[2] T.K. Caughey, Classical normal modes in damped linear dynamic systems, J. Appl. Mech., 42 (1961) 583-588.

[3] R. D. Copetti., J. R. Claeyssen., T. Tsukazan, Modal Formulation of Segmented EulerBernoulli Beams, Math. Prob. Eng., (2007) 18 pages doi:10.1155/2007/36261

[4] J.R. Claeyssen, On predicting the response of non-conservative linear vibrating systems by using dynamical matrix solutions, J. Sound Vib., 140 (1990) 73-84.

[5] J.R. Claeyssen., T. Tsukazan, R. D. Copetti, IMA J. Appl. Math., 78(5) (2013) 1032-1050.

[6] J.R. Claeyssen., T. Tsukazan, R. D. Copetti, Mech. Syst. Sig. Proc., 38 (2013) 299-311.

[7] S.G. Kelly, "Advanced Vibration Analysis", Taylor \& Francis, New York, 2007.

[8] Z. Oniszczuk, Free transverse vibrations of elastically connected simply supported doublebeam complex system, J. Sound Vib., 232(2) (2000) 387-403.

[9] A.B. Seibel, R.D. Copetti, Vibrações livres de uma viga dupla tipo Euler-Bernoulli, em "XXXIV Congresso Nacional de MAtemática Aplicada e Computacional", Águas de Lindóia, pp. 88-89, 2012.

[10] H.V. Vu, Vibration of a double-beam system, J. Sound Vib., 229 (2000) 807-822. 